\section{Supervivencia prolongada en el síndrome de Casamassima-Morton- Nance. Reporte de caso y revisión de la literatura}

\begin{abstract}
RESUMEN
El síndrome de Casamassima-Morton-Nance es una entidad rara, de etiología desconocida y mal pronóstico, que pertenece al grupo heterogéneo de las disostosis espondilocostales. Este síndrome, además de defectos vertebrales y arcos costales, comprende atresia anal y anormalidades genitourinarias como criptorquidia, hidrocele, hidronefrosis, agenesia renal, cloaca y fístulas urinarias, entre otras. En este trabajo informamos el caso de una niña de seis años de edad, sin historia de consanguinidad y con cariotipo normal, que presenta alteraciones congénitas, costovertebrales, anales y urogenitales compatibles con el síndrome de Casamassima-Morton-Nance. En su evolución se agregaron retraso psicomotor grave, epilepsia y litiasis renal recidivante, hallazgos no reportados en los casos previos, posiblemente por el fallecimiento temprano. Se han propuesto como mecanismos causales la herencia autosómica recesiva, mutaciones de novo y al menos dos casos han presentado rearreglos cromosómicos. Este reporte propone la posibilidad de supervivencia hasta la etapa escolar a partir de un abordaje multidisciplinario temprano; aunque el síndrome puede condicionar retraso psicomotor importante. La etiología permanece desconocida y no se descarta que sea heterogénea, por lo que los casos son candidatos a hibridación genómica comparativa en microarreglos o a secuenciación exómica.
\end{abstract}

Palabras clave: síndrome de Casamassima-Morton-Nance, disostosis espondilocostal, malformación urogenital, litiasis renal recidivante, retraso psicomotor.
Nidia Huerta-Uribe ${ }^{1}$

Camilo Villarroel-Cortés ${ }^{2}$

${ }^{1}$ Médico Pediatra.

${ }^{2}$ Médico Especialista en Genética Humana. Departamento de Genética Humana. Instituto Nacional de Pediatría, México, D.F.
Recibido: 15 de abril del 2014

Aceptado: 11 de agosto del 2014

Correspondencia: Nidia Huerta Uribe Insurgentes Sur 3700-C

Col. Insurgentes Cuicuilco CP 04530, México, Distrito Federal Teléfono: 044 (55) 29365073 nidiahu33@hotmail.com

Este artículo debe citarse como Huerta-Uribe N, Villarroel-Cortés C. Supervivencia prolongada en el síndrome de CasamassimaMorton-Nance. Reporte de caso y revisión de la literatura. Acta Pediatr Mex 2015;36:89-96. 
renal agenesis, cloaca and urinary fistula, among others. In this work we report a 6 year-old girl, no history of consanguinity and with normal karyotype, presenting costovertebral, anal and urogenital congenital defects, consistent with CMNS. In the evolution of the disease, the patient presented severe psychomotor delay, epilepsy and relapsing renal lithiasis. These findings have not been mentioned in the previous reports, probably due to short survival. The causal mechanisms proposed are autosomal recessive inheritance, de novo mutations and chromosomal rearrangements, present in at least two cases. This report illustrates the possibility of achieving a longer survival, up to scholar age, by an early multidisciplinary approach, although the syndrome may cause severe psychomotor delay. The aetiology remains unknown, perhaps being heterogeneous, which makes these patients suitable for comparative genomic hybridization to microarrays or exome sequencing.

Key words: Casamassima Morton Nance Syndrome, spondylocostal dysostosis, urogenital malformation, relapsing renal lithiasis, psychomotor delay.

\section{INTRODUCCIÓN}

El síndrome de Casamassima-Morton-Nance fue descrito por primera vez en 1981 en dos hermanos menonitas que presentaban disostosis espondilocostal, atresia anal y anomalías urogenitales. ${ }^{1}$ Este síndrome pertenece al grupo heterogéneo de las disostosis espondilocostales o espondilotorácicas, ${ }^{2}$ en el que los hallazgos en común incluyen anormalidades en la segmentación de las vértebras y arcos costales. ${ }^{3}$ Además de los defectos espondilocostales este síndrome se caracteriza por atresia anal y anormalidades genitourinarias como criptorquidia, hidrocele, hidronefrosis, agenesia renal, cloaca y fístulas urinarias, entre otras.

Aunque la incidencia de los defectos vertebrales congénitos es alta (1 por cada 1000 recién nacidos vivos) ${ }^{4}$ en el caso del síndrome de Casamassima-Morton-Nance sólo hay seis pacientes reportados hasta el momento, por lo que su incidencia real se desconoce; sin embargo, en un estudio retrospectivo en España se estimó una frecuencia de 0.3 por cada 100000 recién nacidos vivos. ${ }^{5}$ Por el antecedente de consanguinidad en el primer reporte se sugirió una etiología genética con patrón de herencia autosómico recesivo, ${ }^{1}$ no sustentada en el resto de los casos reportados. El pronóstico se considera malo debido a que todos los casos reportados, excepto uno, han fallecido de forma temprana o han sido interrumpidos prenatalmente, es por ello que se desconoce mucho de la evolución clínica en los supervivientes.

En este informe presentamos el caso de una niña de seis años de edad con síndrome de Casamassima-Morton-Nance y discutimos los hallazgos clínicos con respecto a lo reportado en la literatura.

\section{REPORTE DE CASO}

Niña de seis años, producto del tercer embarazo de una madre de 24 años y padre de 25 años de edad al nacimiento, ambos de origen mexicano, sanos, sin historia de consanguinidad, endogamia 
o familiares afectados. Embarazo sin contacto con teratógenos, al cuarto mes de gestación se detectó por ultrasonografía hidropesía fetal, hidrometrocolpos y probable ano imperforado, por lo que se refirió a un centro especializado para su seguimiento. Nació por parto eutócico a término, con un peso de $3370 \mathrm{~g}$, talla de $49 \mathrm{~cm}$ y Apgar de 6 requiriendo 2 ciclos de presión positiva.

Al nacimiento se corroboraron la malformación anorrectal con cloaca y ano imperforado, además de hidronefrosis bilateral, persistencia del conducto arterioso y deformidad torácica. El ano imperforado, la hidronefrosis y la cardiopatía congénita hicieron necesario realizar vesicostomía y colostomía descendente, así como cierre quirúrgico de la persistencia del conducto arterioso. La paciente se envió al Instituto Nacional de Pediatría para continuar su manejo.

A la exploración física se encontraron peso y talla por debajo del centil 3, hipotonía generalizada, dolicocefalia, frente amplia, hipertricosis, cejas difusas en tercio medio y externo, puente nasal ancho, úlceras corneales, narinas antevertidas, labio inferior bífido, boca en carpa, paladar íntegro, pabellones auriculares bien conformados, tórax asimétrico, ano imperforado, genitales con hipoplasia importante de labios menores y mayores, clítoris grande, apertura única tipo cloaca, sin extrofia. Escoliosis con convexidad torácica derecha, prominencia ósea a nivel de sacro. Extremidades hipotróficas e hipotónicas, manos y pies sin alteraciones morfológicas.

Dentro de sus exámenes paraclínicos se realizó serie ósea radiológica que demostró 9 arcos costales derechos e izquierdos, con deformidad en los últimos, además de ausencia de dos cuerpos vertebrales y hemivértebras a nivel torácico (Figura 1); serie esófago-gastro-duodenal con alteración de la mecánica de la deglución y reflujo gastroesofágico. Ecocardiograma con hipertensión arterial pulmonar, insuficiencia tricuspídea severa y mitral leve. Ultrasonografía con hidronefrosis persistente (Figura 2) y reflujo vesicoureteral bilateral. Cistouretrografía miccional con vejiga neurogénica con incontinencia urinaria. Colograma distal con paso del medio de contraste del recto sigmoides a la vejiga por comunicación distal (cloaca) (Figura 3).

Durante su evolución cursó con peso y talla bajos y displasia broncopulmonar; actualmente

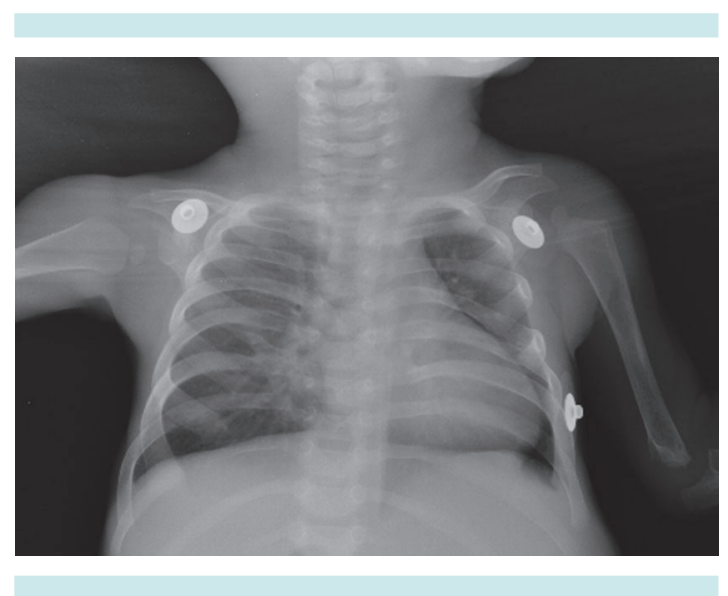

Figura 1. Radiografía posteroanterior de tórax: 9 arcos costales bilaterales con deformidad de arcos costales izquierdos y hemivértebras a nivel torácico.

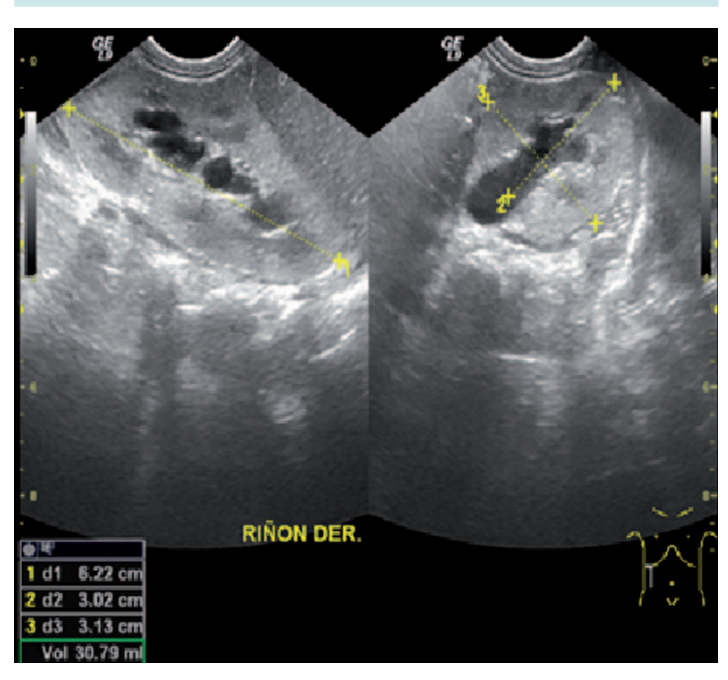

Figura 2. Ultrasonografía renal: hidronefrosis renal derecha. 


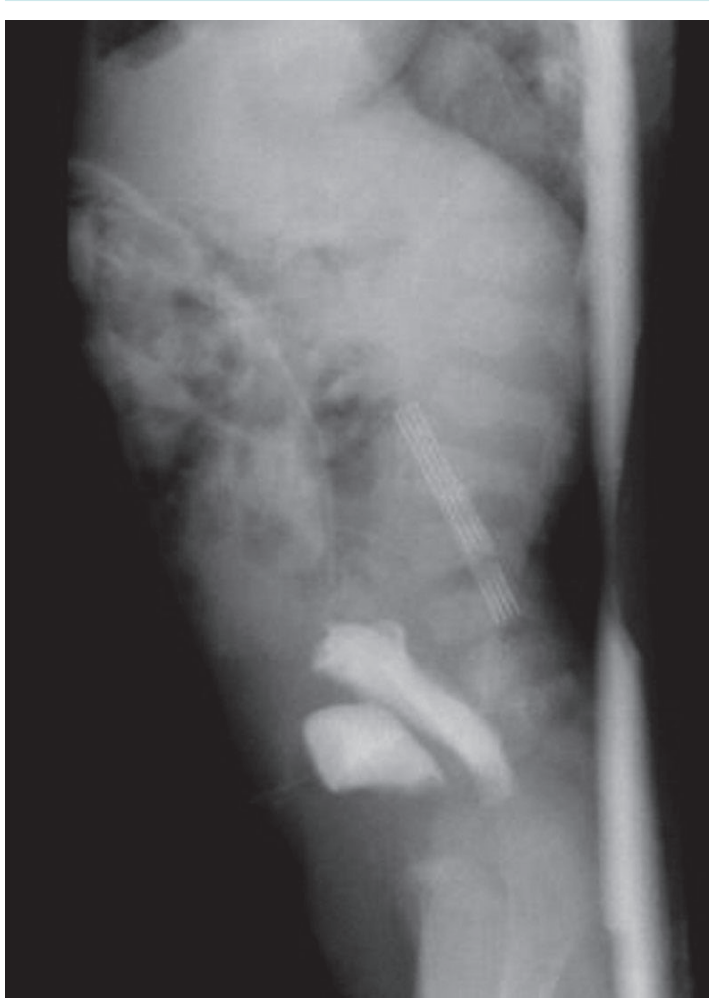

Figura 3. Colograma distal: paso del medio de contraste del recto sigmoides a la vejiga por comunicación distal.

sin requerir oxígeno suplementario. A los 10 meses de edad presentó prolapso de vesicostomía, por lo que se efectuó trigonoplastía y rehechura de vesicostomía. A los 2 años 8 meses de edad presentó litiasis renal y vesical tratada con litotomía. A los 3 años 9 meses cursó con cuadro de obstrucción intestinal y litiasis recidivante, por lo que se practicó laparotomía exploradora con adherenciólisis extensa, corte y ligadura de fístula rectocloacal, drenaje de absceso retro- y suprapúbico y nefrolitotomía con colocación de catéter doble J. Su última hospitalización fue a los 4 años 1 mes de edad, también por litiasis recidivante, incluyendo un lito coraliforme en pelvis renal izquierda, con función renal preservada (Figura 4). Además, la paciente cursó con múltiples hospitalizaciones por procesos infec-

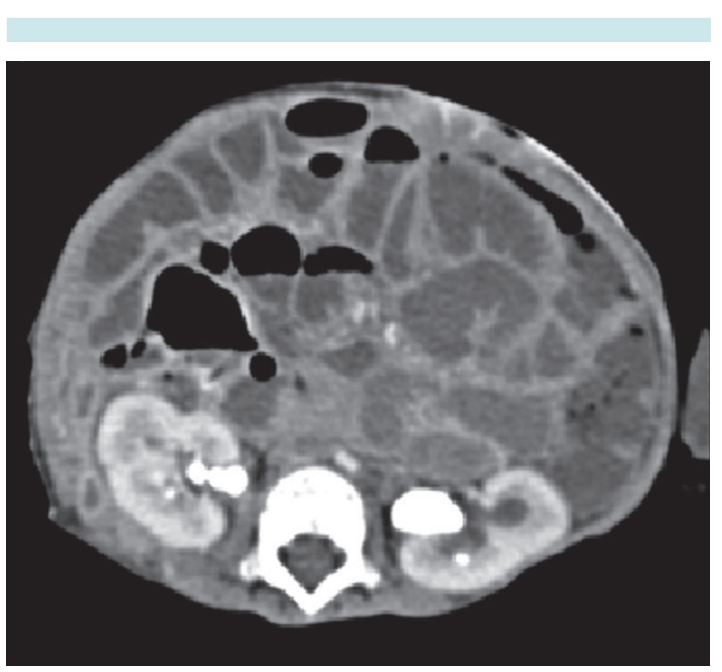

Figura 4. Tomografía axial computada de abdomen: 3 litos renales derechos y 1 lito coraliforme izquierdo que abarca toda la pelvis renal.

ciosos a nivel pulmonar, abdominal y urinario, todos resueltos con éxito.

Sus hitos del desarrollo mostraron un retraso significativo de manera global, presentó fijación de la mirada y sonrisa social a los 2 meses, pinza gruesa a los 2 años, balbuceos a los 2 años 10 meses. Actualmente presenta pobre control de tronco y no ha desarrollado lenguaje. Además del retraso psicomotor grave, la paciente continua con desnutrición crónica.

\section{DISCUSIÓN}

Consideramos que las características encontradas en esta paciente son compatibles con el síndrome de Casamassima-Morton-Nance que se incluye en el catálogo de entidades mendelianas (OMIM 271520). Este síndrome se caracteriza por atresia anal y anormalidades genitourinarias aunadas a disostosis espondilocostal, ${ }^{3}$ todas de presentación grave. La mayoría de los casos, incluyendo el presente, han sido detectados prenatalmente por medio de ultrasonografía de segundo trimestre, lo que sugiere que es una herramienta valiosa en 
la sospecha. Las anomalías encontradas más frecuentemente mediante ultrasonografía incluyen: masas abdominales, hidronefrosis, arteria umbilical única y anormalidades vertebrales. A pesar de la gravedad de los defectos en la mayoría de los casos el crecimiento intrauterino fue normal; sólo en dos casos, con muerte prenatal, se reportaron peso y talla bajos para la edad gestacional. ${ }^{3,6} \mathrm{En}$ cuanto a los hallazgos fenotípicos posnatales todos los casos presentaron hemivértebras y anomalías de arcos costales como fusión, agenesia y costillas bífidas, entre otras. También son constantes las alteraciones anales ya que seis de los siete casos presentaron atresia anal y el restante se reportó con implantación anterior.? Igualmente, todos presentaron malformaciones urogenitales diversas: cuatro casos con hidronefrosis y tres casos, incluyendo el nuestro, con seno urogenital o fístula rectovesical. Dentro de las malformaciones genitales descritas más frecuentemente se encuentran: hidrometrocolpos, vagina septada $y$ agenesia de genitales externos o internos. ${ }^{1-3,6-8}$ Con base en lo anterior consideramos que la tríada de anomalías costovertebrales, anales y genitourinarias se mantiene hasta ahora como el criterio principal para el diagnóstico.

Debemos destacar que en ninguno de los otros casos se reporta litiasis renal, mientras que nuestro caso presentó gran morbilidad asociada con litiasis recidivante. Las causas más frecuentes de este hallazgo en niños incluyen historia o presencia de infección en vías urinarias, antecedentes familiares de litiasis y trastornos metabólicos..$^{9,10}$ En nuestra paciente consideramos que la causa puede ser multifactorial, siendo determinantes la malformación de las vías urinarias e inmovilización, que de forma secundaria provocaron infecciones urinarias recurrentes. Existe riesgo de disfunción y cicatrices renales que se deben vigilar en la paciente.

Los hallazgos de cardiopatía congénita, defectos intestinales y dismorfias faciales son menos constantes. En tres casos, incluyendo el presentado, se reportaron una o más malformaciones cardiacas, entre ellas persistencia del conducto arterioso, comunicación interventricular, doble salida de ventrículo derecho, estenosis de la arteria pulmonar e insuficiencias valvulares. ${ }^{4,8}$ Los defectos intestinales reportados incluyen: vólvulo y malrotación intestinal, divertículo de Meckel y calcificaciones abdominales; ${ }^{2,6,8}$ que no se encontraron en nuestra paciente.

Por otro lado, en la mayoría de los casos descritos no fue posible evaluar el desarrollo psicomotor, principalmente por el fallecimiento temprano; tampoco han sido descritos defectos estructurales del sistema nervioso central. Sin embargo, nuestra paciente tiene retraso psicomotor grave, epilepsia parcial y síndrome hipotónico, lo que hace probable que este síndrome se asocie con estas alteraciones neurológicas y confiera un pronóstico de retraso mental de grave a profundo para los supervivientes, lo cual incrementaría la morbilidad y disminuye la calidad de vida de los afectados.

En el diagnóstico diferencial debemos considerar principalmente al espectro de síndromes con anomalías de la segmentación de las vértebras y costillas: el síndrome de Jarcho-Levin o disostosis espondilocostal 1 (SCDO1) (OMIM 277300) es el más reconocido de ellos; ${ }^{5} \sin$ embargo, tanto éste como SCDO2, SCDO3, SCDO4 y SCDO5, están constituidos únicamente por defectos severos costovertebrales y la presencia de cloaca e hidronefrosis bilateral los excluyen. La asociación VACTERL presenta defectos vertebrales, atresia anal, alteraciones renales y cardiopatía congénita; no obstante, las alteraciones costales, la presencia de cloaca y el retraso psicomotor no forman parte de este diagnóstico. ${ }^{11}$ Por otro lado, se sabe que los pacientes con anemia de Fanconi frecuentemente presentan defectos al nacimiento, que sugieren el diagnóstico de asociación VACTERL, siendo 
las más frecuentes las anomalías radiales y renales ${ }^{11}$ pero sin incluir las alteraciones costales y la cloaca mencionadas.

En cuanto a la forma de herencia y etiología del síndrome, con excepción del primer caso propuesto como probable autosómico recesivo, ${ }^{1}$ ninguno de los otros cuenta con antecedente de consanguinidad o hermanos afectados. Mortier y sus colaboradores ${ }^{12}$ analizaron 26 pacientes con múltiples defectos de la segmentación vertebral y concluyeron que la mayoría de los casos asociados con malformaciones mayores de otros órganos pertenecen a casos esporádicos, dentro de los que se pueden incluir mutaciones de novo. Daïkha-Dahmane y sus colegas ${ }^{8}$ informaron sobre un caso de síndrome de Casamassima-Morton-Nance con una translocación balanceada $(6 ; 9)(p 12 ; q 12)$ heredada de la madre, sugiriendo algún gen responsable en los loci de los puntos de ruptura en $6 \mathrm{p} \mathrm{o}$ 9q. ${ }^{8}$ En 2012, Sasaki y su grupo ${ }^{6}$ reportaron un caso con una deleción de novo (8)(p23.1). Este locus contiene el gen del factor de transcripción GATA4, relacionado con malformaciones cardiacas, pero no con malformaciones urogenitales, anales y espondilocostales. Se sabe que el desarrollo vertebral está controlado por un número limitado de genes, dentro de los que se encuentran DLL3, MESP2, SCDO3, LFNG yHES7, responsables de formas monogénicas de disostosis espondilocostal con herencia autosómica recesiva incluyendo Jarcho-Levin, ${ }^{13}$ y TBX6 responsable de 2 casos de disostosis espondilocostal autosómica dominante. ${ }^{4}$ Sin embargo, tampoco las alteraciones en estos genes explicarían el conjunto de defectos del síndrome de Casamassima-Morton-Nance.

Dada la probable relación del síndrome de Casamassima-Morton-Nance tanto con mutaciones recesivas como con desbalances cromosómicos, además de cariotipo, existe la necesidad de realizar en los afectados tanto el estudio de hibridación genómica comparativa en microarreglos (arrayCGH) como de secuenciación exómica, ${ }^{14-16}$ que podrían permitir la detección de las variantes causales del trastorno. Ambos métodos desafortunadamente son poco accesibles en nuestro medio, por lo que no pudieron realizarse en este caso. Por otro lado, teratógenos como la diabetes materna, radiación ionizante, alcohol y fármacos también se han descrito como perturbadores del proceso de desarrollo embriológico del esqueleto axial, ${ }^{17,18}$ pero en los casos del síndrome de CasamassimaMorton-Nance no ha sido descrito el contacto con dichos agentes. Podemos apreciar entonces que la etiología no está clara, pero que existe una gran probabilidad de una causa heterogénea para el fenotipo del síndrome de CasamassimaMorton-Nance.

La disostosis espondilocostal se asocia con una gran variedad de anomalías congénitas. ${ }^{19}$ En una revisión de otros defectos en niños con disostosis espondilocostal se encontró a la disgenesia caudal como la más frecuentemente asociada, ${ }^{20} \mathrm{y}$ se refiere que la mayoría de los casos con disostosis espondilocostal y anomalías urogenitales, anales u otras, se han considerado erróneamente como Jarcho-Levin (SCDO 1), lo que sugiere que el síndrome de Casamassima-Morton-Nance, además de presentar una baja incidencia, ha sido subdiagnosticado debido en parte a que la clasificación de estas entidades inicialmente era poco específica y a que no se ha definido por completo el espectro clínico del síndrome de Casamassima-Morton-Nance.

La evolución y pronóstico de este síndrome son malos. De los tres casos previos, nacidos vivos, uno murió a los 7 meses de edad, otro se reporta vivo a los 20 meses y otro a los 4 años que, sin embargo, tuvo la presentación más leve sin ano imperforado. ${ }^{1,2,7}$ No existe relación entre el grado de afección vertebral y la letalidad del síndrome, ${ }^{12}$ siendo la gravedad de los defectos 
asociados la que va a determinar el pronóstico. ${ }^{5}$ La mayor morbilidad está asociada con restricción pulmonar, infecciones recurrentes y complicaciones renales secundarias a las malformaciones genitourinarias, así como con los múltiples procedimientos quirúrgicos requeridos para corregir los defectos. ${ }^{2,7}$

Nuestra paciente tiene supervivencia prolongada, probablemente por el abordaje oportuno y la corrección exitosa de las malformaciones presentadas, así como un tratamiento enérgico de las infecciones pulmonares y de vías urinarias.

\section{CONCLUSIONES}

Aunque el síndrome de Casamassima-MortonNance es raro, posiblemente se encuentre subdiagnosticado y tiene gran importancia clínica por tratarse de una entidad multimalformativa con pronóstico en general pobre; los problemas restrictivos a nivel pulmonar y las complicaciones renales secundarias son las causas principales de morbilidad y mortalidad.

Este reporte ilustra la posibilidad de supervivencia hasta la etapa escolar a partir de un abordaje multidisciplinario temprano. Sin embargo, los supervivientes pueden presentar problemas neurológicos importantes como retraso psicomotor grave. La etiología permanece desconocida y no se descarta que sea heterogénea, por lo que los casos son candidatos a estudios moleculares tales como arrayCGH o a secuenciación exómica.

\section{REFERENCIAS}

1. Casamassima AC, Morton CC, Nance WE, Kodroff M, Caldwell R, Kelly T, et al. Spondylocostal Dysostosis Associated with Anal and Urogenital Anomalies in a Mennonite Sibship. Am J Med Genet 1981;8:117-127.
2. Aguinaga $M$, Yllescas E, Canseco M, Machuca A, Acevedo $S$, Mayén-Molina DG. Prenatal Clinical Characteristics of Casamassima-Morton-Nance Syndrome. Prenat Diagn 2009;29:172-176.

3. Thauvin-Robinet. Spondylocostal Dysostosis, Anal and Genitourinary Malformations in a Fetal Case: A New Case of Casamassima-Morton-Nance Syndrome? Eur J Med Genet 2007;50:85-91.

4. Sparrow DB, McInerney-Leo A, Gucev ZS, Gardiner B, Marshall $\mathrm{M}$, Leo PJ, et al. Autosomal Dominant Spondylocostal Dysostosis is Caused by Mutation in TBX6. Hum Mol Genet 2013;22(8):1625-1631.

5. Martínez-Frías ML, Bermejo-Sánchez E, Martínez-Santana S, Nieto-Conde C, Engüés-Jimeno J, Pérez-Fernández JL, et al. Síndromes de Jarcho-Levin y Casamassima: Diagnóstico Diferencial y Frecuencia en España. An Esp Pediatr 1998;48(5):510-514.

6. Sasaki A, Hayashi S, Oi R, Anami A, Hanaoka M, Miyazaki $\mathrm{O}$, et al. A Fetus Diagnosed with Casamassima-MortonNance Syndrome with de novo del(8)(p23.1). Prenat Diagn 2011;31:407-409.

7. Pérez FJ, Tobar V, González LE. Síndrome de Casamassima Morton Nance: Reporte de Caso y Revisión de la Literatura. Urol Colom 2009;18(3):121-126.

8. Daikha-Dahmane F, Huten Y, Morvan J, Szpiro-Tapia S, Nessmann C, Eydoux P. Fetus with Casamassima-MortonNance Syndrome and an Inherited (6;9) Balanced Translocation. Am J Med Genet 1998;80:514-517.

9. Camacho Díaz JA, Casas Gómez J, Amat Barnés A, Giménez Llort A, García García L. Litiasis Renal en el Niño. An Esp Pediatr 1996;44(3):225-228.

10. Santos-Ruiz MI, Hidalgo-Barquero del Rosal E, GarcíaBlanco JM. Urolitiasis en la Infancia: Revisión Clínica y Epidemiológica de los Últimos Años en Nuestro Medio. Vox Pediátrica 2004;12(1):13-19.

11. Alter BP, Rosenberg PS. VACTERL-H Association and Fanconi Anemia. Mol Syndromol 2013;4:87-93.

12. Mortier GR, Lachman RS, Bocian M, Rimoin DL. Multiple Vertebral Segmentation Defects: Analysis of $26 \mathrm{New}$ Patients and Review of the Literature. Am J Med Genet 1996;61:310-319.

13. Gucev ZS, Tasic V, Pop-Jordanova N, Sparrow DB, Dunwoodie SL, Ellard S, et al. Autosomal Dominant Spondylocostal Dysostosis in Three Generations of a Macedonian Family: Negative Mutation Analysis of DLL3, MESP2, HES7, and LFNG. Am J Med Genet Part A 2010;152A:1378-1382.

14. Theisen A. Microarray-based Comparative Genomic Hybridization (aCGH). Nature Education 2008;1(1):45.

15. Bamshad MJ, Ng SB, Bigham AW, Tabor HK, Emond MJ, Nickerson DA, et al. Exom Sequencing as a Tool 
for Mendelian Disease Gene Discovery. Nat Rev Genet 2011;12:745-755.

16. Kiezun A, Garimella K, Do R, Stitziel NO, Neale BM, McLaren PJ, et al. Exome Sequencing and the Genetic Basis of Complex Traits. Nat Genet 2012;44(6):623-630.

17. Nazer J, Ramírez R. Malformaciones Congénitas en los Hijos de Madres Diabéticas. Rev Med Chile [online] 2000;128(9):1045-1052.

18. Chen CP. Syndromes, Disorders and Maternal Risk Factors Associated with Neural Tube Defects (II). Taiwan J Obstet Gynecol 2008;47(1):10-17.
19. Devos EA, Leroy JG, Braeckman JJ, Vanden-Bulcke LJ, Langer LO. Spondylocostal Dysostosis and Urinary Tract Anomaly: Definition and Review of an Entity. Eur J Pediatr 1978;128:7-15.

20. Martínez-Frías ML, Bermejo-Sánchez E, Paisán L, Martín M, Egüés J, López JA. Severe Spondylocostal Dysostosis Associated With Other Congenital Anomalies: A Clinical/Epidemiologic Analysis and Description of Ten Cases From the Spanish Registry. Am J Med Genet 1994;51:203-212. 